\title{
ON URYSOHN-CLOSED AND MINIMAL URYSOHN SPACES
}

\author{
JAMES E. JOSEPH
}

\begin{abstract}
In this paper, several characterizations of Urysohn-closed and minimal Urysohn spaces are given along with some relating properties.
\end{abstract}

1. Introduction. In [2], characterizations of Urysohn-closed and minimal Urysohn spaces have been given in terms of arbitrary filterbases and a type of convergence for filterbases called $u$-convergence. In the present article, we utilize these characterizations to obtain characterizations of these spaces (1) in terms of projections, and (2) in terms of graphs of functions into the space; both of these-graphs and projections-are used together with any class $\mathcal{S}$ of spaces containing as a subclass the Hausdorff completely normal fully normal spaces to effect the characterizations. In the last section, we give some examples. See [1] for a survey of results on Urysohn-closed and minimal Urysohn spaces.

2. Preliminaries. The closure of a subset $K$ of a topological space will be denoted by $\operatorname{cl}(K)$. If $\psi, \lambda: X \rightarrow Y$ are functions, $\{x \in X: \psi(x)=\lambda(x)\}$ will be denoted by $\mathcal{E}(\psi, \lambda, X, Y)$. Let $X$ be a space and let $x \in X$. $G$ and $H$ will be called an ordered pair of open sets about $x$ if $x \in G \subset \operatorname{cl}(G) \subset H$ [2].

Definition 2.1. A point $x$ in a space $X$ is in the $u$-closure of $a$ subset $K$ of the space $(x \in u$-cl $(K))$ if each ordered pair $(G, H)$ of open sets about $x$ satisfies $K \cap \operatorname{cl}(H) \neq \varnothing$.

Definition 2.2. A subset $K$ of a space is $u$-closed if it contains its $u$-closure $(u-\operatorname{cl}(K) \subset K)$.

DEFINITION 2.3. [2]. A point $x$ in a space is in the $u$-adherence of a filterbase $\Omega$ on the space $(x \in u$-ad $\Omega)$ if each $F \in \Omega$ and ordered pair $(G, H)$ of open sets about $x$ satisfy $F \cap \operatorname{cl}(H) \neq \varnothing$.

Definition 2.4 [1]. A space $X$ is Urysohn if for $x, y \in X$ with $x \neq y$, there are open sets $V$ and $W$ about $x$ and $y$, respectively, satisfying $\operatorname{cl}(V) \cap \operatorname{cl}(W)$ $=\varnothing$.

THEOREM 2.5. The following statements are equivalent for a space $X$.

(a) $X$ is Urysohn,

Received by the editors June 24, 1976.

AMS (MOS) subject classifications (1970). Primary 54D25, 54D30; Secondary 54C10.

Key words and phrases. Urysohn-closed spaces, minimal Urysohn spaces, functions with closed graphs.

() American Mathematical Society 1978 
(b) $\{x\}=\bigcap_{\Sigma} u-\operatorname{cl}(V)$ for each $x \in X$ and open set base $\Sigma$ at $x$.

(c) Each point in $X$ is $u$-closed.

We use the following characterization from Theorem 3.2 of [2] as a primitive in this paper.

Definition 2.6. A Urysohn space is Urysohn-closed if each filterbase on the space has nonempty $u$-adherence.

In stating our next two theorems, which may be readily established, we recall that a point $x$ in a space is in the $\theta$-closure of a subset $K(x \in \theta-\operatorname{cl}(K))$ of the space if each $V$ open about $x$ satisfies $K \cap \operatorname{cl}(V) \neq \varnothing$; and a subset $K$ of a space is $\theta$-closed if $\theta-\operatorname{cl}(K) \subset K$ [6]. We further recall from [6] that a point $x$ in a space is in the $\theta$-adherence of a filterbase $\Omega$ on the space $(x \in \theta-\operatorname{ad} \Omega)$ if $x \in \theta-\operatorname{cl}(F)$ for each $F \in \Omega$. In addition, we employ the following definition.

Definition 2.7. If $X$ and $Y$ are spaces, $(x, y) \in X \times Y$ is in the first coordinate u-closure of $K \subset X \times Y((x, y) \in(1) u$-cl $(K))$ if $K \cap(\operatorname{cl}(H) \times V)$ $\neq \varnothing$ for any $V$ open about $y$, and ordered pair $(G, H)$ of open sets about $x$. $K$ is (1) $u$-closed if $(1) u-\operatorname{cl}(K) \subset K$.

THEOREM 2.8. The following statements hold for topological spaces $X$ and $Y$, subsets $K$ and $M$ of $X$ and subsets $P$ and $Q$ of $X \times Y$.

(a) $X(X \times Y)$ and $\varnothing$ are $u$-closed $((1) u$-closed $)$.

(b) $K \subset \operatorname{cl}(K) \subset \theta-\operatorname{cl}(K) \subset u-\operatorname{cl}(K) ; \quad P \subset \operatorname{cl}(P) \subset(1) u-\operatorname{cl}(P) \subset u$-cl $(P)$; $u$-closed ((1)u-closed) subsets are $\theta$-closed (closed).

(c) The intersection of any collection and union of any finite collection of $u$-closed $((1) u$-closed $)$ subsets of $X(X \times Y)$ are $u$-closed $((1) u$-closed $)$.

(d) If $K \subset M(P \subset Q)$, then $u-\operatorname{cl}(K) \subset u-\operatorname{cl}(M)((1) u-\operatorname{cl}(P) \subset(1) u-\operatorname{cl}(Q))$.

(e) We have $u$-ad $\Omega=\cap_{\Omega} u$-cl $(F)$ and $\theta$-ad $\Omega \subset u$-ad $\Omega$ for each filterbase $\Omega$ on $X$.

It is known that a function $\psi: X \rightarrow Y$ is continuous if $\psi(\operatorname{ad} \Omega) \subset \operatorname{ad} \psi(\Omega)$ for each filterbase $\Omega$ on $X$. A function $\psi: X \rightarrow Y$ is weakly-continuous if for each $x \in X$ and $U$ open about $\psi(x)$, there is a $V$ open about $x$ satisfying $\psi(V) \subset \operatorname{cl}(U)[5]$. We remark that the following result is valid.

THEOREM 2.9. A function $\psi: X \rightarrow Y$ is weakly-continuous if and only if $\psi(\operatorname{ad} \Omega) \subset \theta$-ad $\psi(\Omega)$ for each filterbase $\Omega$ on $X$.

These observations motivate the following definition.

Definition 2.10. A function $\psi: X \rightarrow Y$ is $u$-weakly-continuous if $\psi(\operatorname{ad} \Omega) \subset$ $u$-ad $\psi(\Omega)$ for each filterbase $\Omega$ on $X$.

Theorem 2.11 gives various characterizations of $u$-weakly-continuous functions.

THEOREM 2.11. The following statements are equivalent for spaces $X, Y$ and function $\psi: X \rightarrow Y$.

(a) $\psi$ is $u$-weakly-continuous.

(b) $\psi(\operatorname{cl}(K)) \subset u$-cl $(\psi(K))$ for each $K \subset X$. 
(c) For each $x \in X$ and ordered pair $(G, H)$ of open sets about $\psi(x)$, there is $a V$ open about $x$ satisfying $\psi(V) \subset \operatorname{cl}(H)$.

In [3], a function $\psi: X \rightarrow Y$ is said to have a strongly-closed graph if for each $(x, y) \in(X \times Y)-G(\psi)$, there is a $V$ open about $x$ and $U$ open about $y$ satisfying $(V \times \operatorname{cl}(U)) \cap G(\psi)=\varnothing$. It is not difficult to show that, for a $T_{2}$ space $Y$, this condition is equivalent to the condition $\theta-\operatorname{ad} \psi(\Omega) \subset\{\psi(x)\}$ for each $x \in X$ and filterbase $\Omega$ on $X-\{x\}$ with $\Omega \rightarrow x$. This characterization motivates Definition 2.12.

Definition 2.12. A function $\psi: X \rightarrow Y$ has a u-strongly-subclosed graph if $u$-ad $\psi(\Omega) \cup\{\psi(x)\}=\{\psi(x)\}$ for each $x \in X$ and filterbase $\Omega$ on $X-\{x\}$ with $\Omega \rightarrow x$.

Definition 2.13. Let $X$ be a set, let $x_{0} \in X$, and let $\Omega$ be a filterbase on $X$; $\left\{A \subset X: x_{0} \in X-A\right.$ or $F \cup\left\{x_{0}\right\} \subset A$ for some $\left.F \in \Omega\right\}$ is a topology on $X$ which will be called the topology on $X$ associated with $x_{0}$ and $\Omega . X$ equipped with this topology will be called the space associated with $x_{0}$ and $\Omega$. We will denote this space by $X\left(x_{0}, \Omega\right)$.

The following easily established theorem is used frequently in the sequel.

Theorem 2.14. Let $X$ be a set, let $x_{0} \in X$ and let $\Omega$ be a filterbase on $X$ which has empty intersection on $X-\left\{x_{0}\right\}$. Then $X\left(x_{0}, \Omega\right)$ is in class $\delta$.

3. Characterizations of Urysohn-closed spaces by functions with $\boldsymbol{u}$-stronglysubclosed graphs. In [3], it is proved that a Hausdorff space $Y$ is $H$-closed if and only if all functions with strongly-closed graphs from a space in class $\mathfrak{S}$ to $Y$ are weakly-continuous. The first of our main theorems is an analogue of this result for Urysohn-closed spaces. In the proof of sufficiency in each of the theorems below, it is easily checked from Theorem 2.14 that the space associated with the indicated point and filterbase is in class $\mathcal{S}$.

THEOREM 3.1. A Urysohn space $Y$ is Urysohn-closed if and only if for each space $X$ in class $\mathcal{S}$, each bijection $\psi: X \rightarrow Y$ with a u-strongly-subclosed graph is $u$-weakly-continuous.

Proof. Necessity. Let $Y$ be a Urysohn-closed space, let $X$ be any space and let $\psi: X \rightarrow Y$ be any function with a $u$-strongly-subclosed graph. Let $\Omega$ be a filterbase on $X$ and let $y \in \psi(\operatorname{ad} \Omega)$. Choose $x \in \operatorname{ad} \Omega$ with $\psi(x)=y$ and let $\Sigma$ be an open set base at $x$. Let $\Omega^{*}=\{(V \cap F)-\{x\}: V \in \Sigma, F \in \Omega\}$. If $\Omega^{*}$ is not a filterbase on $X$, we have $V \cap F=\{x\}$ for some $V \in \Sigma$ and $F \in \Omega$; so $x \in F$ for each $F \in \Omega$. This gives $\psi(x) \in \psi(F)$ for each $F \in \Omega$. If $\Omega^{*}$ is a filterbase on $X$, then $\Omega^{*}$ is a filterbase on $X-\{x\}$ and $\Omega^{*} \rightarrow x$. Furthermore, $\psi\left(\Omega^{*}\right)$ is a filterbase on $Y$; since $Y$ is Urysohn-closed and $\psi$ has a $u$-strongly-subclosed graph, we get $\{\psi(x)\}=u$-ad $\psi\left(\Omega^{*}\right) \subset u$-ad $\psi(\Omega)$.

Sufficiency. Let $\Omega$ be a filterbase on the Urysohn space $Y$ and suppose $u$-ad $\Omega \cap\left(Y-\left\{y_{0}\right\}\right)=\varnothing$ for some $y_{0} \in Y$. Let $\psi: Y\left(y_{0}, \Omega\right) \rightarrow Y$ be the identity function. We show that $\psi$ has a $u$-strongly-subclosed graph. Let $y \in Y\left(y_{0}, \Omega\right)$ and let $\Omega^{*}$ be a filterbase on $Y\left(y_{0}, \Omega\right)-\{y\}$ such that $\Omega^{*} \rightarrow y$. 
It is clear that $y$ must be $y_{0}$. It follows also that for each $F \in \Omega$, there is an $F^{*} \in \Omega^{*}$ such that $F^{*} \subset F$. So, if $v \in u$-ad $\Omega^{*}$, we must have $v \in u$-ad $\Omega$. Thus, $u$-ad $\Omega^{*} \cup\left\{y_{0}\right\}=\left\{y_{0}\right\}$ and $\psi$ has a $u$-strongly-subclosed graph. We now have that $\psi$ is $u$-weakly-continuous if $Y$ satisfies the condition, so $y_{0} \in \operatorname{ad} \Omega=\psi(\operatorname{ad} \Omega) \subset u-\operatorname{ad} \psi(\Omega)=u-\operatorname{ad} \Omega$.

The proof is complete.

THEOREM 3.2. A Urysohn space $Y$ is Urysohn-closed if and only if for each space $X$ in class $\mathcal{S}$ and bijections $\psi, \lambda: X \rightarrow Y$ with $u$-strongly-subclosed graphs, $\mathcal{E}(\psi, \lambda, X, Y)$ is closed in $X$.

Proof. Necessity. Let $Y$ be Urysohn-closed, let $X$ be any space and let $\psi, \lambda$ : $X \rightarrow Y$ be any functions with $u$-strongly-subclosed graphs; $\psi$ is $u$-weaklycontinuous from Theorem 3.1. Let $x \in \operatorname{cl}(\mathcal{E}(\psi, \lambda, X, Y))-\mathcal{E}(\psi, \lambda, X, Y)$. There is a filterbase $\Omega$ on $\delta(\psi, \lambda, X, Y)$ with $\Omega \rightarrow x$. Since $\lambda$ has a $u$ strongly-subclosed graph, we have

and

$$
\{\psi(x)\} \cup\{\lambda(x)\} \subset \psi(\operatorname{ad} \Omega) \cup\{\lambda(x)\} \subset u-\operatorname{ad} \psi(\Omega) \cup\{\lambda(x)\}
$$

$$
u-\operatorname{ad} \psi(\Omega)=u-\operatorname{ad} \lambda(\Omega)
$$

a contradiction.

Sufficiency. Let $\Omega$ be a filterbase on the Urysohn space $Y$ and suppose $u$-ad $\Omega=\varnothing$. Choose $x_{0}, y_{0} \in Y$ with $x_{0} \neq y_{0}$. Let $\psi: Y\left(y_{0}, \Omega\right) \rightarrow Y$ be the identity function; define $\lambda: Y\left(y_{0}, \Omega\right) \rightarrow Y$ by $\lambda\left(x_{0}\right)=y_{0}, \lambda\left(y_{0}\right)=x_{0}$ and $\lambda(x)=x$ otherwise. We see that $\psi$ and $\lambda$ are bijections and that $\psi$ has a $u$-strongly-subclosed graph as in the proof of the sufficiency of Theorem 3.1. We show that $\lambda$ has a $u$-strongly-subclosed graph. Let $y \in Y$ and let $\Omega^{*}$ be a filterbase on $Y\left(y_{0}, \Omega\right)-\{y\}$ with $\Omega^{*} \rightarrow y$. Then, $y=y_{0}$; so, for each $F \in \Omega$, there is an $F^{*} \in \Omega^{*}$ with $F^{*} \subset F$. Thus, there is an $F_{0}^{*} \in \Omega^{*}$ with $x_{0} \notin F_{0}^{*}$. Let $\Omega^{* *}=\left\{F^{*} \cap F_{0}^{*}: F^{*} \in \Omega^{*}\right\}$. Then, $u$-ad $\lambda\left(\Omega^{*}\right)=u$-ad $\lambda\left(\Omega^{* *}\right)=u$ ad $\Omega^{* *} \subset u$-ad $\Omega$. This establishes that $\lambda$ has a $u$-strongly-subclosed graph; however, $\mathcal{E}\left(\psi, \lambda, Y\left(y_{0}, \Omega\right), Y\right)=Y-\left\{x_{0}, y_{0}\right\}$, which is not closed in $Y\left(y_{0}, \Omega\right)$.

The proof is complete.

THEOREM 3.3. A Urysohn space $Y$ is Urysohn-closed if and only if for each space $X$ in class $\mathcal{S}$ and functions $\psi, \lambda: X \rightarrow Y$ (one of these a bijection) with $u$-strongly-subclosed graphs, $\mathcal{E}(\psi, \lambda, X, Y)=X$ whenever $\mathcal{E}(\psi, \lambda, X, Y)$ is dense in $X$.

Proof. Necessity. Let $Y$ be Urysohn-closed, let $X$ be any space and let $\psi, \lambda$ : $X \rightarrow Y$ be any functions with $u$-strongly-subclosed graphs. If $\mathcal{E}(\psi, \lambda, X, Y)$ is dense in $X$, then $\mathcal{E}(\psi, \lambda, X, Y)=X$ since $\mathcal{E}(\psi, \lambda, X, Y)$ is closed in $X$ from Theorem 3.2.

Sufficiency. We follow the proof of the sufficiency in Theorem 3.2 to the point immediately preceding the definition of $\lambda$. We define $\lambda$ by $\lambda(x)=x$ if 
$x \neq y_{0}$ and $\lambda\left(y_{0}\right)=x_{0}$. Arguments similar to those in the proof of the sufficiency of Theorem 3.2 show that $\psi$ and $\lambda$ have $u$-strongly-subclosed graphs. $\mathcal{E}\left(\psi, \lambda, Y\left(y_{0}, \Omega\right), Y\right)=Y-\left\{y_{0}\right\}$ which is dense in $Y\left(y_{0}, \Omega\right)$, a contradiction. The proof is complete.

4. Characterizations of Urysohn-closed spaces by projections. In [4] it is proved that a Hausdorff space $X$ is $H$-closed if and only if the projection $\pi_{y}$ : $X \times Y \rightarrow Y$ takes $\theta$-closed subsets onto $\theta$-closed subsets for every space $Y$ in class $\mathcal{S}$. Although we have not been able to establish that a Urysohn space is Urysohn-closed if and only if $\pi_{y}: X \times Y \rightarrow Y$ maps $u$-closed subsets onto $u$-closed subsets of $Y$ for every space $Y$ in class $\mathcal{S}$, we can give two characterizations of Urysohn-closed spaces in terms of projections. Theorem 4.3 is given without proof.

THEOREM 4.1. A Urysohn space $X$ is Urysohn-closed if and only if $\pi_{y}$ : $X \times Y \rightarrow Y$ maps (1)u-closed subsets of $X \times Y$ onto closed subsets of $Y$ for every space $Y$ in class $\mathcal{S}$.

Proof. Necessity. Let $X$ be Urysohn-closed and let $Y$ be any space. Let $K \subset X \times Y$ be (1)u-closed and let $y \in \operatorname{cl}\left(\pi_{y}(K)\right) . \quad\left\{\pi_{x}((X \times V) \cap K): V\right.$ open about $y$ \} is a filterbase on $X$ and has a $u$-adherent point, $x$. If $V$ is open about $y$ and $(G, H)$ is an ordered pair of open sets about $x$, then $\operatorname{cl}(H) \cap$ $\pi_{x}((X \times V) \cap K) \neq \varnothing$; so $(\operatorname{cl}(H) \times V) \cap K \neq \varnothing$ and $(x, y) \in(1) u$-cl $(K)$ $\subset K$. Thus, $y \in \pi_{y}(K)$.

Sufficiency. Let $\Omega$ be a filterbase on the Urysohn space $X$ with $u$-ad $\Omega=\varnothing$. Let $y_{0} \notin X$, let $Y=X \cup\left\{y_{0}\right\}$, and let $K=\{(x, x): x \in X\}$. We show that $K$ is (1) u-closed in $X \times Y\left(y_{0}, \Omega\right)$. Let $(m, n) \in\left(X \times Y\left(y_{0}, \Omega\right)\right)-K$. Then $m \neq n$ and $m \neq y_{0}$. Since $X$ is Urysohn, there is an ordered pair $(G(1), H(1))$ of open sets about $m$ such that $n \notin \operatorname{cl}(H(1))$. Also, since $u$-ad $\Omega=\varnothing$, there is an $F \in \Omega$ and an ordered pair $(G(2), H(2))$ of open sets about $m$ satisfying $F \cap \operatorname{cl}(H(2))=\varnothing$. Let $G=G(1) \cap G(2)$ and $H=H(1) \cap H(2)$. Then $(G, H)$ is an ordered pair of open sets for $m$, and $F \cup\left\{n, y_{0}\right\} \subset Y-\operatorname{cl}_{X}(H)$. Now, we have $Y-\operatorname{cl}_{X}(H)$ open about $n$ in $Y\left(y_{0}, \Omega\right)$, and $(\operatorname{cl}(H) \times(Y-$ $\left.\left.\operatorname{cl}_{X}(H)\right)\right) \cap K=\varnothing$ for the pair $(G, H)$. Consequently, $(m, n) \notin(1) u$-cl $(K)$ and $K$ is (1)u-closed; $\pi_{y}(K)$ is therefore closed in $Y\left(y_{0}, \Omega\right)$ and $y_{0} \in$ $\operatorname{cl}\left(\pi_{y}(K)\right)$. This contradiction completes the proof.

Definition 4.2. Let $X$ and $Y$ be spaces; $(x, y) \in X \times Y$ is in the pre-uclosure, of $K \subset X \times Y((x, y) \in$ pre-u-cl $(K))$ if each ordered pair $(G, H)$ of open subsets about $(x, y)$ with $H$ a basic open set satisfies $K \cap \operatorname{cl}(H) \neq \varnothing$. $K$ is pre-u-closed if pre-u-cl( $K) \subset K$.

THEOREM 4.3. A Urysohn space $X$ is Urysohn-closed if and only if $\pi_{y}$ : $X \times Y \rightarrow Y$ maps pre-u-closed subsets of $X \times Y$ onto $u$-closed subsets of $Y$ for every space $Y$ in class $\mathcal{S}$.

5. Characterizations of minimal Urysohn spaces by graphs. In [2], minimal Urysohn spaces have been characterized as precisely those Urysohn spaces in 
which each filterbase with at most one $u$-adherent point is convergent. In this section, we offer several other characterizations of minimal Urysohn spaces.

THEOREM 5.1. A Urysohn space $Y$ is minimal Urysohn if and only if for each space $X$ in class $\mathcal{S}$, each bijection $\psi: X \rightarrow Y$ with a u-strongly-subclosed graph is continuous.

Proof. Necessity. Let $Y$ be minimal Urysohn, let $X$ be any space, let $\psi$ : $X \rightarrow Y$ be any function with a $u$-strongly-subclosed graph, and let $x \in X$. If $\{x\}$ is open in $X, \psi$ is continuous at $x$. If not, then $\Omega=\{V-\{x\}: V$ open about $x\}$ is a filterbase on $X-\{x\}$ and $\Omega \rightarrow x$. So, $u$-ad $\psi(\Omega)=\varnothing$ or $\{\psi(x)\}$ since $\psi$ has a $u$-strongly-subclosed graph. Therefore, $\psi(\Omega) \rightarrow \psi(x)$ and for each $U$ open about $\psi(x)$, there is a $V$ open about $x$ with $\psi(V) \subset U$.

Sufficiency. Let $\Omega$ be a filterbase on $Y$ with at most one $u$-adherent point, $y_{0}$, in $Y$. If $\Omega \nrightarrow y_{0}$, there is an open set $V_{0}$ about $y_{0}$ with $\Omega^{*}=\{F \cap(Y-$ $\left.\left.V_{0}\right): F \in \Omega\right\}$ a filterbase on $Y$. If $\psi: Y\left(y_{0}, \Omega^{*}\right) \rightarrow Y$ is the identity function and $\Omega^{* *}$ is a filterbase on $Y\left(y_{0}, \Omega^{*}\right)-\{y\}$ such that $\Omega^{* *} \rightarrow y$, it follows that $y=y_{0}$, and that $u$-ad $\Omega^{* *} \subset u$-ad $\Omega^{*} \subset u$-ad $\Omega$. So, $\psi$ has a $u$-stronglysubclosed graph and is continuous if $Y$ satisfies the condition. Therefore, we have $\Omega \rightarrow y_{0}$ in $Y$ since $\Omega \rightarrow y_{0}$ in $Y\left(y_{0}, \Omega^{*}\right)$.

The proof is complete.

THEOREM 5.2. A Urysohn space $Y$ is minimal Urysohn if and only if for each space $X$ in class $\mathcal{S}$ and bijections $\lambda, \psi: X \rightarrow Y$ with closed graph and $u$ strongly-subclosed graph, respectively, $\mathcal{E}(\psi, \lambda, X, Y)$ is closed in $X$.

Proof. Necessity. Let $Y$ be minimal Urysohn, let $X$ be any space and let $\psi$, $\lambda: X \rightarrow Y$ be any functions with $u$-strongly-subclosed and closed graph, respectively. Since $\psi$ has a $u$-strongly-subclosed graph, $\psi$ is continuous, and the restriction to $G(\psi)$ of the projection $\pi_{x}: X \times Y \rightarrow X$ is a homeomorphism. $G(\psi) \cap G(\lambda)$ is a closed subset of $G(\psi)$, so $\mathcal{E}(\psi, \lambda, X, Y)$ $=\pi_{x}(G(\psi) \cap G(\lambda))$ is closed in $X$.

Sufficiency. Let $\Omega$ be a filterbase on $Y$ with at most one $u$-adherent point, $y_{0}$, in $Y$. If $\Omega \nrightarrow y_{0}$, there is an open set $V_{0}$ about $y_{0}$ with $\Omega^{*}=\{F \cap(Y-$ $\left.\left.V_{0}\right): F \in \Omega\right\}$ a filterbase on $Y$. Choose $x_{0} \in Y-\left\{y_{0}\right\}$, let $\psi: Y\left(y_{0}, \Omega^{*}\right) \rightarrow Y$ be the identity function, and let $\lambda: Y\left(y_{0}, \Omega^{*}\right) \rightarrow Y$ be defined by $\lambda\left(x_{0}\right)=y_{0}$, $\lambda\left(y_{0}\right)=x_{0}$, and $\lambda(x)=x$ otherwise. We see easily that $\delta\left(\psi, \lambda, Y\left(y_{0}, \Omega^{*}\right), Y\right)$ $=Y-\left\{x_{0}, y_{0}\right\}$, which is not closed in $Y\left(y_{0}, \Omega^{*}\right)$. We show that $\psi$ has a $u$-strongly-subclosed graph and that $\lambda$ has a closed graph, a contradiction, completing the proof if $Y$ satisfies the condition;

(a) $\psi$ has a u-strongly-subclosed graph. See proof of sufficiency in Theorem 5.1 ;

(b) $\lambda$ has a closed graph. Let $y \in Y$ and let $\Omega^{* *}$ be a filterbase on $Y\left(y_{0}, \Omega^{*}\right)$ with $\Omega^{* *} \rightarrow y$. Then $y=y_{0}$, and for each $F \in \Omega$ there is an $F^{* *} \in \Omega^{* *}$ satisfying $F^{* *} \subset F \cap\left(Y-V_{0}\right)$. We may assume, without loss, as the demonstration in the proof of the sufficiency of Theorem 3.2 verifies, that 
$\Omega^{* *}$ is a filterbase on $Y-\left\{x_{0}, y_{0}\right\}$. Thus, ad $\lambda\left(\Omega^{* *}\right)=\operatorname{ad} \Omega^{* *}$, ad $\Omega^{* *} \subset$ ad $\Omega^{*}=\varnothing$ and, consequently, $\lambda$ has a closed graph.

The proof is complete.

THEOREM 5.3. A Urysohn space $Y$ is minimal Urysohn if and only if for each space $X$ in class $\mathcal{S}$, and functions (one of these a bijection) $\psi, \lambda: X \rightarrow Y$ with $u$-strongly-subclosed graph and closed graph, respectively, $\mathcal{E}(\psi, \lambda, X, Y)=X$ whenever $\mathcal{E}(\psi, \lambda, X, Y)$ is dense in $X$.

Proof. Parallels that of Theorem 3.3.

REMARK 5.4. In each of the proofs in the theorems of $\$ \$ 3,4$ and 5 we have proved significantly more than the proof of the necessity requires. For example in Theorem 3.1 the proof of the necessity requires that we show for each space $X$ in class $\mathcal{S}$ that each bijection $\psi: X \rightarrow Y$ with a $u$-strongly-subclosed graph is $u$-weakly-continuous. We have established that this statement is valid for any space $X$ and any function $\psi: X \rightarrow Y$.

6. First countable Urysohn spaces. See [1] for definitions and results used but not given here. Noting that $X\left(x_{0}, \Omega\right)$ is metrizable when $\Omega=\left\{F_{n}\right\}$ is countable and has empty intersection on $X-\left\{x_{0}\right\}\left(X\left(x_{0}, \Omega\right)\right.$ is regular and $\{\mathscr{V}(n)\}$ defined by

$$
\mathscr{V}(n)=\left\{F_{n} \cup\left\{x_{0}\right\}\right\} \cup\left\{\{x\}: x \in X-\left(F_{n} \cup\left\{x_{0}\right\}\right)\right\}
$$

is a $\sigma$-locally finite base), we may establish the following theorems by appropriate use of the first countability and arguments similar to those in the last section.

THEOREM 6.1. The following statements are equivalent for a first countable Urysohn space $Y . Q$ may represent the class of first countable spaces, the class of first countable spaces in class $\mathcal{S}$, or the class of metric spaces.

(a) Each countable filterbase on $Y$ has nonvoid $u$-adherence.

(b) Each sequence in $Y u$-accumulates to some point in $Y$.

(c) For each $X \in \mathcal{Q}$, each function (bijection) $\psi: X \rightarrow Y$ with a u-stronglysubclosed graph is u-weakly-continuous.

(d) For each $X \in \mathbb{Q}$ and any two functions (bijections) $\psi, \lambda: X \rightarrow Y$ with $u$-strongly-subclosed graphs, $\mathcal{E}(\psi, \lambda, X, Y)$ is closed in $X$.

(e) For each $X \in \mathbb{Q}$ and any two functions (one of these a bijection) $\psi, \lambda$ : $X \rightarrow Y$ with u-strongly-subclosed graphs, $\delta(\psi, \lambda, X, Y)=X$ when $\mathcal{E}(\psi, \lambda, X, Y)$ is dense in $X$.

THEOREM 6.2. The following statements are equivalent for a first countable Urysohn space $Y . Q$ is as in Theorem 6.1.

(a) Each countable filterbase on $Y$ with at most one $u$-adherent point is convergent.

(b) Each sequence in $Y$ with at most one u-accumulation point converges.

(c) For each $X \in Q$, each function (bijection) $\psi: X \rightarrow Y$ with a u-stronglysubclosed graph is continuous. 
(d) For each $X \in \mathbb{Q}$ and any two functions (bijections) $\psi, \lambda: X \rightarrow Y$ with $u$-strongly-subclosed graph and closed graph, respectively, $\mathcal{E}(\psi, \lambda, X, Y)$ is closed in $X$.

(e) For each $X \in \mathbb{Q}$ and any two functions (one a bijection) $\psi, \lambda: X \rightarrow Y$ with $u$-strongly-subclosed graph and closed graph, respectively, $\mathcal{E}(\psi, \lambda, X, Y)=X$ whenever $\mathcal{E}(\psi, \lambda, X, Y)$ is dense in $X$.

COROllary 6.3. A first countable Urysohn space $Y$ is first countable and Urysohn-closed (minimal Urysohn) if $Y$ satisfies any of the equivalent statements of Theorem 6.1 (6.2).

Proof. Follows from Theorem 4.2 (4.4) of [2].

7. Some examples. In this section, we give some examples to indicate some of the limitations on the weakening of hypotheses in the theorems in this paper. Let $Y$ be the minimal Urysohn space in [1] which is not compact. Then $Y$ is not minimal Hausdorff and, consequently, not $H$-closed. Let $y_{0} \in Y$ and $\Omega$ be a filterbase on $Y$ satisfying $y_{0} \notin u$-ad $\Omega$ and $\theta$-ad $\Omega=\varnothing$. Choose a $V_{0}$ open in $Y$ about $y_{0}$ such that $\Omega^{*}=\left\{F \cap\left(Y-V_{0}\right): F \in \Omega\right\}$ is a filterbase on $Y$. Choose $x_{0} \in Y-\left\{y_{0}\right\}$, and define $\alpha, \psi, \lambda: Y\left(y_{0}, \Omega^{*}\right) \rightarrow Y$ by $\psi(x)=x$ for all $x, \alpha\left(y_{0}\right)=x_{0}$ and $\alpha(x)=x$ otherwise, $\lambda\left(x_{0}\right)=y_{0}, \lambda\left(y_{0}\right)=x_{0}$ and $\lambda(x)=x$ otherwise.

EXAMPLES 7.1. The phrase, " $u$-strongly-subclosed" cannot be replaced by "strongly-closed" in either of Theorems 3.1, 3.2, 3.3, 5.1, 5.2, or 5.3 even if the phrase "closed graph" is repalced by the phrase "strongly-closed graph." The functions $\alpha, \psi, \lambda$, all have strongly-closed graphs but $\psi$ is not u-weaklycontinuous, $\mathcal{E}\left(\psi, \lambda, Y\left(y_{0}, \Omega^{*}\right), Y\right)=Y-\left\{y_{0}, x_{0}\right\}$, which is not closed in $Y\left(y_{0}, \Omega^{*}\right)$, and $\delta\left(\psi, \alpha, Y\left(y_{0}, \Omega^{*}\right), Y\right)=Y-\left\{y_{0}\right\}$, which is dense in $Y\left(y_{0}, \Omega^{*}\right)$.

\section{REFERENCES}

1. M. P. Berri, J. R. Porter and R. M. Stephenson, Jr., A survey of minimal topological spaces, General Topology and its Relations to Modern Analysis and Algebra. III, Academia, Prague, 1971, pp. 93-114. MR 43 \#3985.

2. L. L. Herrington, Characterizations of Urysohn-closed spaces, Proc. Amer. Math. Soc. 55 (1976), 435-439.

3. L. L. Herrington and P. E. Long, Characterizations of $H$-closed spaces, Proc. Amer. Math. Soc. 48 (1975), 469-475.

4. J. E. Joseph, On H-closed spaces, Proc. Amer. Math. Soc. 55 (1976), 223-226.

5. Norman Levine, $A$ decomposition of continuity in topological spaces, Amer. Math. Monthly 68 (1961), 44-46. MR 23 \# A3548.

6. N. V. Velichko, H-closed topological spaces, Mat. Sb. 70 (112) (1966), 98-112; English transl., Amer. Math. Soc. Transl. (2) 78 (1969), 103-118. MR 33 \#6572.

Department of Mathematics, Federal City College, Washington, D. C. 20001

Current address: Department of Mathematics, Howard University, Washington, D. C. 20059 